\title{
Design, Electrostatic and Eigen Frequency Analysis of Fixed- Fixed Beam MEMS Resonator
}

\author{
Deepak Kumar ${ }^{1}$, A. Vimala Juliet ${ }^{2}$ \\ ${ }_{1,2}^{2}$ (Department of Instrumentation \& Control Engineering / SRM University, India)
}

\begin{abstract}
The objective of this paper is to design of fixed-fixed MEMS Resonator. The beam of resonator is made up of poly-silicon. This paper also presents the electrostatic and Eigen frequency analysis of MEMS resonator. The resonator used in oscillator which can be fabricated on chip. It can also be used as Switch. The size of crystal oscillator is in order of centimeter but the size of MEMS Resonator is in order of microns, so by replace a crystal oscillator by MEMS Resonator, we can reduce the size of the system. The dc voltage is given to the beam of resonator for electrostatic analysis \& Eigen frequency analysis in different modes.
\end{abstract}

Keywords - MEMS Resonator, fixed-fixed beam, mechanical restoring force;

\section{INTRODUCTION}

Micro-Electro-Mechanical Systems (MEMS) are a latest technology in area of mechanical, electrical, electronics and chemical engineering. Micro-Electro-Mechanical Systems, or MEMS, consists of mechanical, electrical systems whose order of size in microns. It is a technology used to miniaturize systems. Electrical components such as inductors and tunable capacitors can be improved significantly compared to their integrated counterparts if they are made using MEMS and Nanotechnology [7]. Resonator is a system that having resonant behavior, it oscillates at some frequencies, called its resonant frequencies. MEMS Resonator is normally used in analog systems, communication systems, switches etc. MEMS resonators are basically time based generators or references whose operating principle is similar to the mechanical tuning fork which is used to tune musical instruments [7]. The oscillations in a resonator can be either electromechanically or electromagnetic. MEMS Resonator have high quality factor as compared to crystal oscillator. Crystal oscillator is bulky and cannot be integrated in IC technology [6]. This paper presents a fixed-fixed beam MEMS Resonator. The resonating beam is fixed at both end, called Anchor that is why called fixed-fixed Resonator. In this paper, fixed-fixed beam resonator is modeled by COMSOL Multiphysics software. The MEMS resonator is designed on silicon-on insulator (SOI). A lot of modes of vibration can be found in a rectangular plate resonator either by changing the thickness of electrodes or by changing the mode number.

\section{PRINCIPLE}

The principle of operation of MEMS Resonator based upon a two electrodes of a capacitor. One electrode is fixed and other is moving called Resonator beam. When an voltage is applied between a fixed-fixed beam and the pull-down electrode, an electrostatic force is induced on the beam. Due to the Electrostatic force the beam will bend and beam will oscillates by applying ac signal at fixed electrode. The resonance frequency is given by [2], [1], [3]

$$
f_{0}=1.03 \sqrt{\frac{E}{\rho}} \frac{h}{L^{2}}
$$

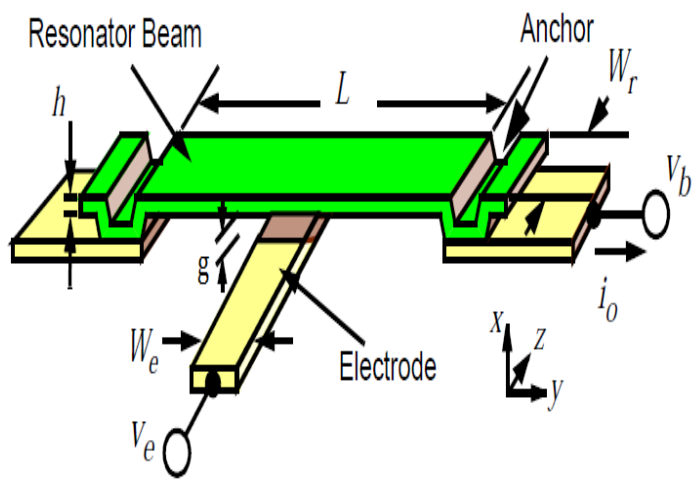

Fig. 1. Fixed-Fixed beam resonator 
Suppose $V_{e}$ is the voltage applied to the electrode and $V_{b}$ is the voltage applied to the beam, then the effective voltage $\left(\mathrm{V}_{\mathrm{e}}-\mathrm{V}_{\mathrm{b}}\right)$ is applied across the electrode to resonator capacitor gap, due to this a force will induced on a beam and amount of force is [4]

$$
F_{b}=\frac{\partial E}{\partial X}=\frac{1}{2}\left(V_{e}-V_{b}\right)^{2} \frac{\partial C}{\partial X}
$$

Where $\mathrm{x}$ is the displacement and $\partial C$ is the change in capacitance between electrodes.

The electrostatic force which is on the plates of a capacitor under an applied voltage is modelled as a parallelplate capacitor. Given that A is the effective area of the beam, which is overlapped by pull-down electrode, the parallel plate Capacitance is [5]

$$
C=\frac{\varepsilon_{0}}{g} A
$$

Where, $\mathrm{g}$ is the height of the beam above the electrode. Energy stored in electrode which act as parallel plate capacitor is

$$
E=\frac{1}{2} C V^{2}
$$

The electrostatic force act on a resonator beam is given by [5]

$$
\mathrm{F}_{\mathrm{e}}=\frac{1}{2} \mathrm{~V}^{2} \frac{d C(g)}{d g}=-\frac{1}{2} \frac{\varepsilon_{0} A V^{2}}{g^{2}}
$$

Where, $\mathrm{V}$ is the Voltage applied between the beam and the electrode.

Equating the applied electrostatic force with the mechanical restoring force due to the stiffness of the beam $(\mathrm{F}=$ $\mathrm{k} \mathrm{x}$ ), we find [5]

$$
\frac{1}{2} \frac{\varepsilon_{0} A V^{2}}{g^{2}}=k\left(g_{0}-g\right)
$$

where, $g_{0}$ is the zero-bias bridge's height. Solving this equation for the voltage results in

The spring constant $\mathrm{K}$ of a fixed-fixed beam is [5]

$$
V=\sqrt{\frac{2 k g^{2}\left(g_{0}-g\right)}{\varepsilon_{0} A}}
$$

Where, E is Young's modulus

$$
K=32 E w\left(\frac{h}{L}\right)^{3}
$$

$\mathrm{h}$ is the thickness of a beam

$\mathrm{L}$ is the length of a beam

$\mathrm{w}$ is the width of a beam .

The condition for which the resonator beam will be stable under electrostatic force is given by

By solving the equation we get

$$
\frac{d V}{d g}=0
$$

$$
g=\frac{2}{3} g_{0}
$$

This is a result of the beam position becoming unstable at $(2 / 3) g_{0}$, which is due to positive feedback in the electrostatic actuation. At $(2 / 3) g_{0}$ the increase in the electrostatic force is greater than the increase in the restoring force, resulting in a beam position becoming unstable and collapse of the beam to the down-state position. The pull-down voltage is found to be

$$
V_{p}=\sqrt{\frac{8 k \mathrm{gg}_{0}{ }^{3}}{27 \varepsilon_{0} A}}
$$

\section{ANALYSIS}

The analysis of MEMS Resonator is done by using software COMSOL Multiphysics. The Resonator has some dimensions which are given in table I.

The Materials used in MEMS resonator:

- Poly Silicon (Electrode)

- $\quad$ Silicon Nitride (Insulator)

- Air (Dielectric)

The poly-silicon material has a varied number of properties which are given in table II.

Fig. 2 shows the design of a MEMS Resonator. The model was introduced in air as a dielectric. 
TABLE 1. Dimension of model

\begin{tabular}{|l|l|}
\hline Dimension & Value \\
\hline Length of beam ( L) & $50 \mu \mathrm{m}$ \\
\hline Width of beam (W) & $10 \mu \mathrm{m}$ \\
\hline Thickness of beam (h) & $2 \mu \mathrm{m}$ \\
\hline Gap spacing (g) & $8 \mu \mathrm{m}$ \\
\hline
\end{tabular}

\subsection{Electrostatic Analysis}

In electrostatic analysis of MEMS Resonator, dc voltage is given to the beam and fixed electrode is kept as a ground. As a voltage is applied the electric field is generated which puts a force on a beam. The pulldown voltage is given by equation (9)

\subsection{Eigen Frequency Analysis}

$$
V_{p}=\sqrt{\frac{8 k g_{0}^{3}}{27 \varepsilon_{0} A}}
$$

In Eigen frequency analysis there are 6 modes of frequencies. The lowest frequency for which these stationary waves are formed is called fundamental harmonic. The modes of oscillation have different shapes for different frequencies. The Fundamental frequencies is given by

(Length) $\mathrm{L}=50 \mu \mathrm{m}$

$$
f_{0}=1.03 \sqrt{\frac{E}{\rho}} \frac{h}{L^{2}}
$$

(Thickness) $\mathrm{h}=2 \mu \mathrm{m}$

$\mathrm{E}=160 \times 10^{9} \mathrm{~Pa}$

$\rho=2320 \mathrm{Kg} / \mathrm{m}^{3}$

$\mathrm{f}_{0}=1.03\left[160 \times 10^{9} / 2320\right]^{-2} \times 2 \times 10^{-6} /\left(50 \times 10^{-6}\right)^{2}$

$=6.84 \mathrm{MHz}$

But the simulated frequency is $7.09 \mathrm{MHz}$.

TABLE 2. Properties of Material

\begin{tabular}{|c|c|}
\hline Property & Value \\
\hline Relative Permittivity & 4.5 \\
\hline Density $(\rho)$ & $2320 \mathrm{~kg} / \mathrm{m}^{3}$ \\
\hline Young's modulus (E) & $160 \times 10^{9} P_{a}$ \\
\hline Poisson's Ratio & 0.22 \\
\hline
\end{tabular}

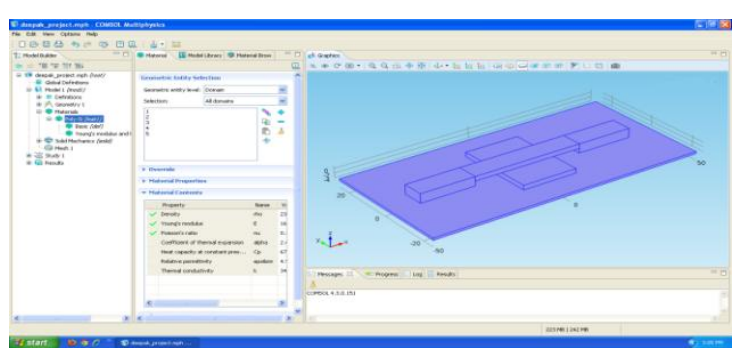

Fig. 2. Design of Resonator

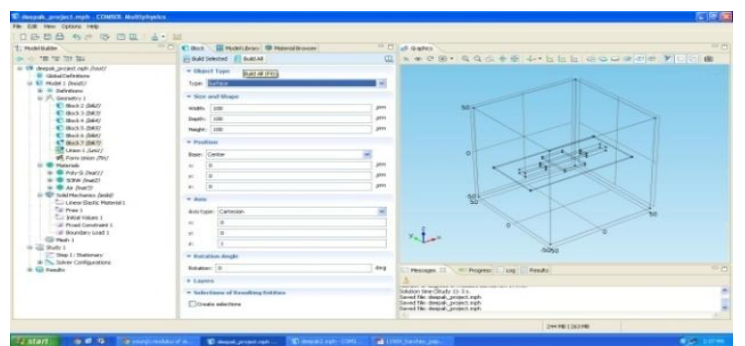

Fig. 3. Model Introduce in Air Dielectric

\subsection{Analysis using COMSOL Multiphysics}

For Eigen frequency analysis, the physics included for the analysis were Electrostatic (es), Solid Mechanics (solid) and study included for the analysis were Eigen Frequency Analysis. The figure shows a simulation results for all modes.

Fig. 4. shows Resonator Beam oscillates Sinusoidal having maximum displacement in middle.

Fig. 5. shows $1^{\text {st }}$ half of the resonator beam oscillates sinusoidal and $2^{\text {nd }}$ half oscillates opposite to the $1^{\text {st }}$.

Fig. 6. Shows $1^{\text {st }}$ Front half of the resonator beam oscillates sinusoidal and $2^{\text {nd }}$ Back half oscillates opposite to the $1^{\text {st }}$.

Fig. 7. Shows only half part of the Resonator Beam oscillates Sinusoidal.

Fig. 8. Shows Resonator Beam oscillates sinusoidal in 3 parts $.1^{\text {st }}$ and $2^{\text {nd }}$ oscillates opposite to the $2^{\text {nd }}$ part.

Fig. 9. Shows $1^{\text {st }}$ half of the resonator beam oscillates same as mode 2 and $2^{\text {nd }}$ half oscillates opposite to the $1^{\text {st }}$. 
TABLE 3. Frequencies of different mode

\begin{tabular}{|c|c|}
\hline Modes & Frequency \\
\hline Mode 1 & $7.09 \mathrm{MHz}$ \\
\hline Mode 2 & $30.08 \mathrm{MHz}$ \\
\hline Mode 3 & $34.37 \mathrm{MHz}$ \\
\hline Mode 4 & $35.63 \mathrm{MHz}$ \\
\hline Mode 5 & $59.16 \mathrm{MHz}$ \\
\hline Mode 6 & $71.55 \mathrm{MHz}$ \\
\hline
\end{tabular}

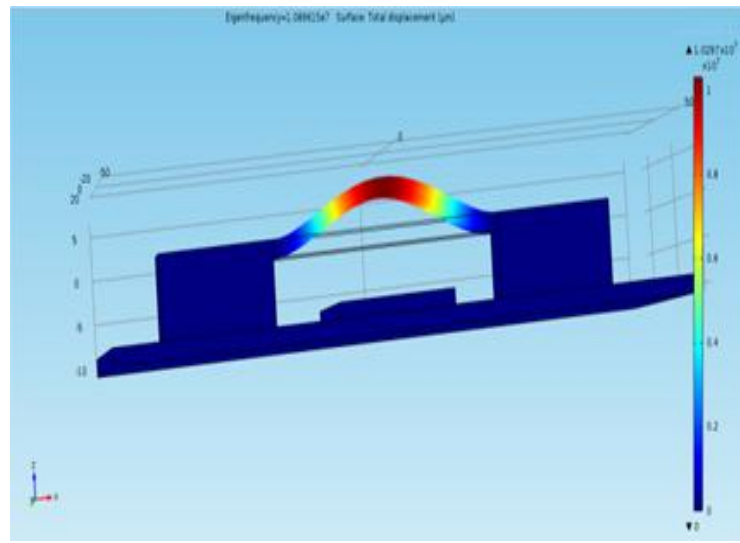

Fig. 4. Mode $1, \mathrm{f}_{0}=7.09 \mathrm{MHz}$

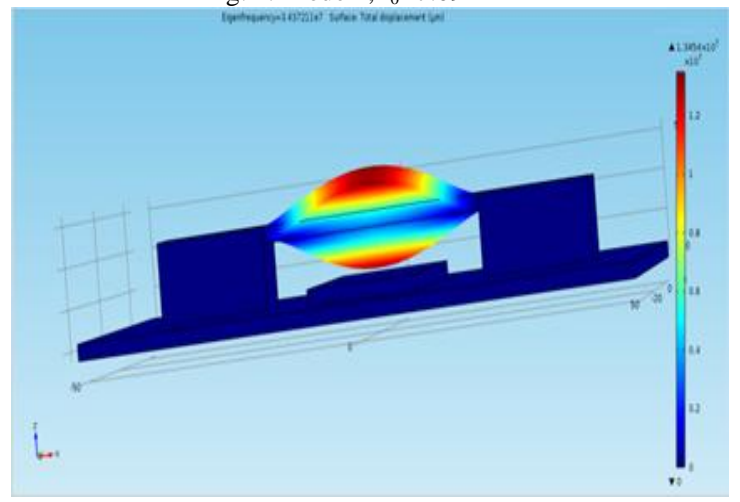

Fig. 6. Mode $3, \mathrm{f}_{0}=34.37 \mathrm{MHz}$

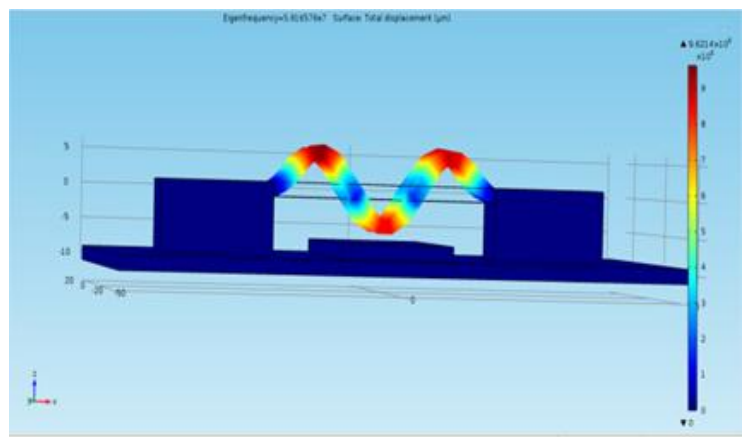

Fig. 8. Mode 5, $\mathrm{f}_{0}=59.16 \mathrm{MHz}$

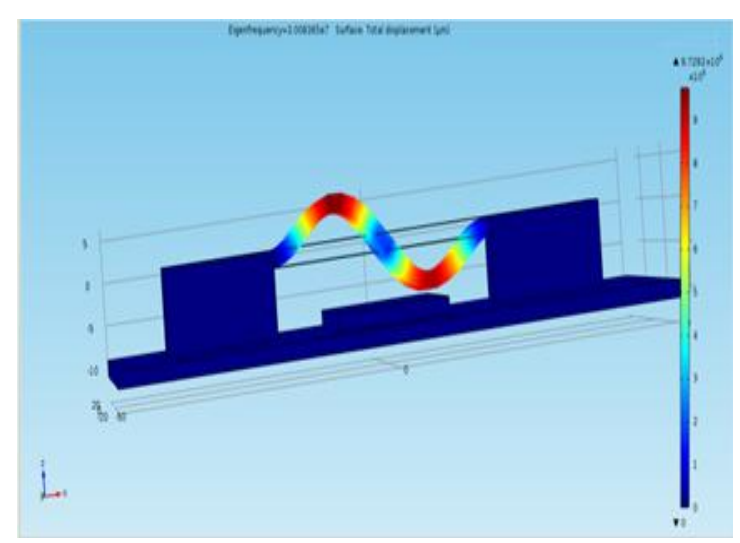

Fig. 5 . Mode $2, \mathrm{f}_{0}=30.08 \mathrm{MHz}$

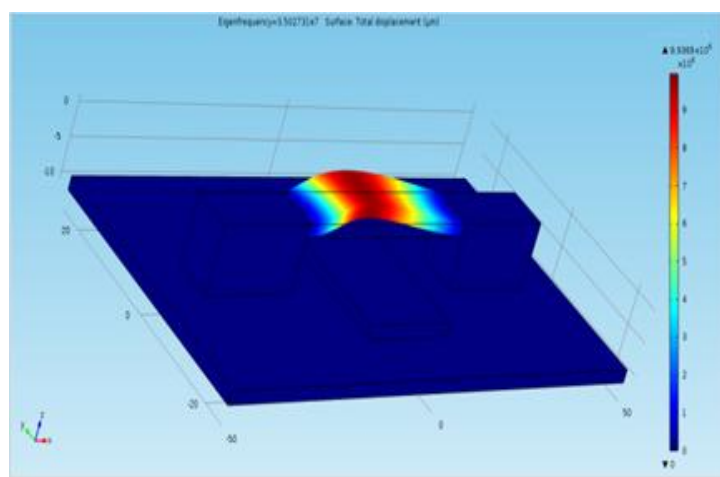

Fig. 7. Mode $4, \mathrm{f}_{0}=35.03 \mathrm{MHz}$

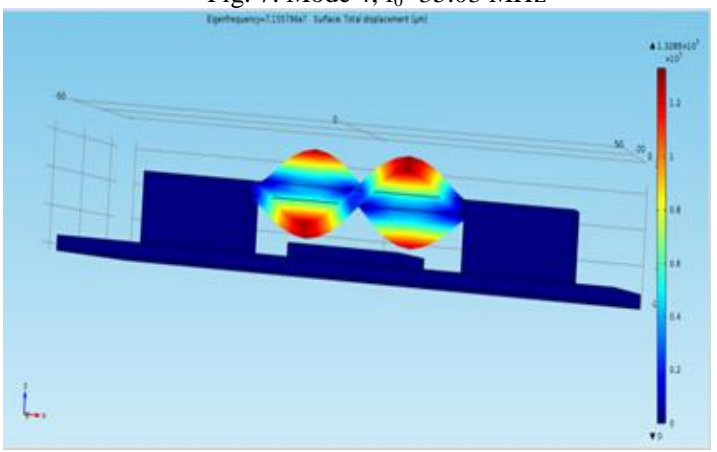

Fig. 9. Mode 6, $\mathrm{f}_{0}=71.55 \mathrm{MHz}$

\section{CONCLUSION}

In simulation, we analyzed the Eigen frequencies of fixed-fixed resonator beam. There are six possible modes of frequencies. $1^{\text {st }}$ mode has fundamental frequency $7.09 \mathrm{MHz}$, also we get different modes with higher frequencies. This method is not limited to fixed-fixed MEMS resonator, this analysis can also be done in other models of MEMS Resonator. Poly-silicon MEMS resonators have been demonstrated up to $71.55 \mathrm{MHz}$ frequencies 


\section{REFERENCES}

[1] Frederic Nabki, Karim Allidina, Faisal Ahmad, Paul-Vahe Cicek, Mourad N. El Gamal, "Highly Integrated 1.8 GHz Frequency Synthesizer Based on a MEMS Resonator" IEEE Journal of solid-state circuits, Vol. 44, No. 8, August 2009, 2154-2168.

[2] G.Meenakshi sundaram, Mahesh Angira, Kamaljit Rangra "Design and Simulation of two port RF MEMS Resonator" International Conference on Computer Communication and Informatics (ICCCI -2012), Jan. 10 - 12, 2012, Coimbatore, INDIA

[3] Vivek Harshey, Amol Morankar, Dr. R.M. Patrikar "MEMS Resonator for RF Applications" Proceeding of the 2011 COMSOL Conference in Banglore, INDIA.

[4] C. T.-C. Nguyen, "Vibrating RF MEMS for low power communications (invited)," Proceedings, 2002 MRS Fall Meeting, Boston,Massachusetts, Dec. 2-6, 2002, pp. J12.1.1-J2.1.12.

[5] Gabriel M.Rebeiz "RF MEMS Theory, Design and Technology" John Wiley and Sons Ltd.

[6] Joep Jacques Marie Bontemps "Design of a MEMS-based $52 \mathrm{MHz}$ Oscillator" Universiteitsdrukkerij Technische Universiteit Eindhoven, The Netherlands-2009

[7] Ravi Patni, Mohit Joshi, Sameer Mehra, and Akhilesh Mohan "Design of Piezoelectric Aluminum Nitride MEMS Resonator"Proceedings of the World Congress on Engineering and Computer Science 2011 Vol I WCECS 2011, October 19-21, 2011, San Francisco, USA. 